\title{
Risk perception, mental health distress, and flourishing during the COVID-19 pandemic in China: The role of positive and negative affect
}

\author{
Ning Zhang ${ }^{1}$ (D) \\ Accepted: 9 December 2021 \\ (c) The Author(s), under exclusive licence to Springer Science+Business Media, LLC, part of Springer Nature 2021
}

\begin{abstract}
The COVID-19 pandemic has brought unprecedented disruptions to people's everyday life and induced wide-ranging impacts on people's physical health, mental health and well-being. This research investigated the relationship between risk perception, mental health distress, and flourishing during the peak period of the COVID-19 pandemic in China. Three hundred and ninety Chinese completed measures on risk perception, mental health distress, positive and negative affect, flourishing, and demographic information. The results revealed that $27.2 \%$ of participants experienced some level of mental health distress, but they also experienced a relatively high level of flourishing. Higher level of risk perception and negative affect were risk factors, whereas positive affect was a protective factor, of mental illness and flourishing. Experiences of positive and negative affect mediated the relationship between risk perception and level of mental health distress and flourishing, respectively. Although the COVID-19 pandemic led to a higher level of mental distress among the general public in China, most people were also resilient during the pandemic. The results have implications for improving mental health and enhancing resiliency during public health crises such as the COVID-19 pandemic.
\end{abstract}

Keywords Risk perception $\cdot$ Positive affect $\cdot$ Negative affect $\cdot$ Mental health $\cdot$ Flourishing

\section{Introduction}

The COVID-19 pandemic, which has led to more than 243 million infections and 4.94 million deaths around the world by October 22nd, 2021, is the most severe public health crisis that human beings are facing since the establishment of the World Health Organization (John Hopkins University, 2020). The high rates of transmission of the COVID-19 virus and the quick spread of misconceptions and rumours about COVID-19 on social media during the early stage of the pandemic induced heightened risk perception - perceived risk of infection during the COVID-19 pandemic - among people around the world (Ioraf et al., 2020; Wise et al., 2020; Zhang et al., 2021). As similar pandemics happened during the past several decades, such as SARS (Maunder

Ning Zhang

zhangning2019@zju.edu.cn

1 School of Public Health and the Second Affiliated Hospital of Zhejiang University School of Medicine, Medical Building, Zhejiang University Zijinggang Campus, Yuhangtang R. 866, Hangzhou 310058, China et al., 2003; Maunder et al., 2006) and Ebola (Lötsch et al., 2017), the COVID-19 pandemic and the associated containing measures (e.g., social distancing, quarantine) not only posed great challenges to people's physical health but also led to wide-ranging impacts on people's mental health and well-being (Brooks et al., 2020; Qiu et al., 2020; Tan et al., 2020; Tian et al., 2020; Xiang et al., 2020; Zhang et al., 2020). For example, a nationwide survey in the early stage of the pandemic in China found that about $35 \%$ of people experienced psychological distress (Qiu et al., 2020). Another study conducted in Singapore found that the prevalence of anxiety, depression, and PTSD was $14.5 \%, 8.9 \%$, and $7.7 \%$, respectively, among healthcare workers (Tan et al., 2020). The research also highlighted that nonmedical healthcare professionals were more prone to psychological distress than medical personnel during the COVID-19 pandemic (Tan et al., 2020). Research conducted during the COVID19 pandemic also revealed that risk perception was a significant predictor of mental health among both healthcare professionals and the general public around the world (Han et al., 2021; Iorfa et al., 2020; Li \& Lyu, 2021; Liu et al., 2021; Yıldırım et al., 2020). 
Despite mounting research on the impacts of the COVID-19 pandemic on mental health distress such as anxiety, depression, stress, and PTSD (Qiu et al., 2020; Tan et al., 2020; Tian et al., 2020), there is a dearth of research on positive mental health or a comprehensive evaluation of the health impacts of the COVID-19 pandemic among the general public. During the past few years, VanderWeele and colleagues (VanderWeele, 2017; VanderWeele et al., 2019) from the Harvard School of Public Health advocated for a comprehensive measure of health to capture what's really important for people's health and well-being. They developed the flourishing measure as a comprehensive index of health and well-being, including dimensions such as physical and mental health, happiness and life satisfaction, meaning and purpose, character and virtue, close social relationships, and financial stability (VanderWeele, 2017; VanderWeele et al., 2019). In response to this advocate, Weziak-Białowolska et al. (2019) have already compared experience of flourishing among people from different cultural backgrounds, showing that the flourishing measure is applicable as a comprehensive measure of health across cultures. In a recent review on the advancement of research on subjective well-being during the past several decades, Gan (2020) advocated researchers to move beyond the view of medical interventions in improving well-being by taking a public health perspective. In the current research, we took this lead to evaluate the mental health impacts of the pandemic among Chinese using both traditional mental health measure such as the General Health Questionnaire (Goldberg et al., 1997; Goldberg \& Hillier, 1979; Goldberg \& Williams, 1988), and the flourishing measure to gain a comprehensive understanding of people's mental health status during the peak period of the COVID-19 pandemic in China.

One of the protective factors of mental health that received accumulating support during the past several decades is that experience of positive emotions can buffer people from negative impacts of stressful events or crises (Folkman \& Moskowitz, 2000; Fredrickson et al., 2003). For example, Fredrickson et al. (2003) found that positive emotions mediated the relationship between precrisis resilience and the development of depressive symptoms after the 9.11 attack among college students in U.S. Besides, positive emotions accounted for the relationship between precrisis and postcrisis growth in psychological resources (Fredrickson et al., 2003). According to the broaden-andbuild theory of positive emotions proposed by Frederickson (Fredrickson, 1998, 2001, 2004), it is likely that those who experienced a higher level of positive emotions would adapt better during the pandemic. Therefore, we hypothesized that experiences of positive versus negative emotions would mediate the relationship between risk perception, mental health issues and experience of flourishing during the COVID-19 pandemic.

\section{Materials and Methods}

\section{Participants}

Three-hundred and ninety-one respondents from 31 provinces and one special administrative area (SAR) of China participated in this study. One participant reported birth year in 2017 was excluded from further analyses, leaving 390 participants in the final sample. The age of respondents ranges from 17 to $69\left(M_{\text {age }}=33.28\right.$ years, $\left.S D=10.26\right)$. Most of the participants are female (69.5\%). About half (49.7\%) of the participants are married and another half are unmarried $(48.2 \%)$, with $1.8 \%$ of them are divorced and $0.3 \%$ of them are widowed. The participants are relatively well-educated, $96.1 \%$ of them have a bachelor's degree or above. Most of the participants belong to Han ethnicity $(94.1 \%)$, and the remaining $5.9 \%$ belong to ethnic minorities.

\section{Measures and Procedures}

The research team conducted this study through an online survey platform (www.wjx.com, which is similar to Qualtrics) in China by distributing the survey link through social media. Participants consented to participate in the survey completed a battery of measures including, risk perception of COVID-19, self-efficacy in coping with COVID-19, General Health Questionnaire, Positive and Negative Affect Schedule, the Flourishing Measure, and questions on demographic information.

\section{Risk Perception and Self-Efficacy}

We measured people's perceived risk of COVID-19 with the following four items adapted from previous research (de Zwart et al., 2009): (1) 'How severe do you think the COVID-19 pandemic is?', $(1=$ not severe at all, $5=$ very severe); (2) 'How do you perceive the health risk of COVID19?'; (3) 'How do you perceive your risk of being infected with COVID-19?'; (4) 'How do you perceive your risk of infection compared to a normal Chinese with the same age and gender to you?', $(1=$ very low risk, $5=$ very high risk $)$. The reliability of risk perception measure in this study is .78. We then measured people' self-efficacy in preventing themselves from being infected with COVID-19 with the following two items: (1) 'How do you perceive your ability of preventing yourself from being infected with COVID19 ?' $(1=$ very good, $5=$ very bad $)$; (2) 'How do you perceive your ability of preventing yourself from being infected with COVID-19 compared to a Chinese with the same age 
and gender to you?', $1=$ much better, $5=$ much worse) . We reverse-coded the items such that a higher score indicating a higher level of self-efficacy in preventing oneself from being infected with COVID-19. The reliability for self-efficacy measure in this study is .89 .

\section{General Health Questionnaire}

The 12-item General Health Questionnaire was developed by Goldberg and colleagues (Goldberg et al., 1997; Goldberg \& Hillier, 1979; Goldberg \& Williams, 1988) to measure the severity of mental health problems during the past few weeks. It has been validated across different cultural contexts as a measure of mental health distress. It has two scoring methods: the GHQ method and the Likert method. By using the GHQ method, participants' responses were coded as $0-0-1-1$, with a sum score ranges from 0 to 12 . A cuttingoff score of 3 was used as a criterion of diagnosing mental illness (Goldberg et al., 1997; Xu et al., 2013). By using the Likert scoring method - a 4-point Likert-type scale (from 0 to 3), participants' responses were added up to generate a total score ranging from 0 to 36 . A higher score indicates a higher severity of mental health issues. As suggested by Banks et al. (1980), the Likert scoring method is more appropriate for parametric multivariate analyses. Therefore, we used the Likert scoring method in the current study. The reliability of the General Health Questionnaire-12 in the current study is good (Cronbach's $\alpha=.87$ ).

\section{Positive and Negative Affect Schedule}

The Positive and Negative Affect Schedule (PANAS) was developed by Watson et al. (1988). PANAS is a self-report measure composed of two mood scales, one measuring positive affect and the other measuring negative affect. The scale consists of twenty words (e.g., interested, distressed) describing positive and negative feelings. Participants indicated the extent to which he/she felt this way over the past week on a five-point scale $(1=$ Very slightly or not at all, $2=$ A little, $3=$ Moderately, $4=$ Quite a bit, $5=$ Extremely). The scale was translated into Chinese and verified for its psychometric properties by Qiu et al. (2008). Cronbach's $\alpha$ for positive affect and negative affect is .93 and .92, respectively, in this study.

\section{Flourishing Measure}

The flourishing measure was developed by VanderWeele and colleagues (VanderWeele, 2017; VanderWeele et al., 2019; Weziak-Białowolska et al., 2019) as a comprehensive measure of health, including happiness and life satisfaction, physical and mental health, meaning and purpose, character and virtue, close social relationships, and financial stability.
Each domain has two items (e.g., 'Overall, how satisfied are you with life as a whole these days?', 'In general, how would you rate your physical health?'). Participants respond to the items on an 11-point Likert scale with the label tailored to each of the items (e.g., $1=$ Not satisfied at all, 10=Completely satisfied, or $1=$ Poor, $10=$ Excellent). Average of participants' responses to the first 10 items is treated as the flourishing index and average of the whole scale is labelled as the secure flourishing index (VanderWeele, 2017; VanderWeele et al., 2019). The scale has been used in crosscultural settings for measuring and comparing human flourishing across countries (Weziak-Białowolska et al., 2019). Cronbach's $\alpha$ for both the flourishing index and the secure flourishing index is .93 in this study. As the results are consistent for the analyses with both the flourishing index and the secure flourishing index as the dependent variable, in the results section we reported all the results based on the secure flourishing index.

Finally, participants answered question on demographic information such as age, gender, marital status, education level, etc. The research received ethical approval from the author's host institution.

\section{Results}

\section{Descriptive Analyses and Correlations among Study Variables}

First, we estimated the prevalence of mental health distress by using the cut-off score of 3 as recommended by previous researchers based on the GHQ scoring method (Goldberg et al., 1997; Xu et al., 2013). The results indicated that about 27.2\% participants scored 3 or higher on the General Health Questionnaire, suggesting that they were experiencing some level of mental health distress during the peak period of the COVID-19 pandemic in China. We then explored the correlations between key variables in the current study. The results revealed that age was negatively correlated with level of risk perception, experience of negative affect, and severity of mental health problems ( $r s<-.13$, ps $<.01)$, but positively correlated with self-efficacy in preventing oneself from infection, experience of positive affect, and flourishing ( $r s \geq .25, p s<.01$ ). Participants' level of risk perception was negatively correlated with their self-efficacy in preventing themselves from infection, experience of positive affect, and flourishing $(r s<-.20, p s<.01)$, but positively associated with experience of negative affect and severity of mental health problems $(r s>.26, p s<.01)$. Self-reported efficacy in preventing oneself from infection was negatively correlated with experience of negative affect and severity of mental health problems $(r s<-.19, p s<.01)$, but positively 
Table 1 Correlations between key variables

\begin{tabular}{|c|c|c|c|c|c|c|c|c|}
\hline \multirow[b]{2}{*}{ Variables } & \multirow[b]{2}{*}{$M$} & \multirow[b]{2}{*}{$S D$} & \multicolumn{6}{|c|}{ Correlation } \\
\hline & & & 1 & 2 & 3 & 4 & 5 & 6 \\
\hline 1.Age & 33.28 & 10.36 & - & & & & & \\
\hline 2.Risk Perception & 2.49 & .67 & $-.17 * *$ & - & & & & \\
\hline 3.Self efficacy & 3.90 & .73 & $.25^{* *}$ & $-.376^{* *}$ & - & & & \\
\hline 4.Positive affect & 3.10 & .88 & $.327 * *$ & $-.203 * *$ & $.41 * *$ & - & & \\
\hline 5.Negative affect & 2.16 & .88 & $-.218 * *$ & $.32 * *$ & $-.199 * *$ & $-.106^{*}$ & - & \\
\hline 6.GHQ & 1.92 & 2.28 & $-.139 * *$ & $.269 * *$ & $-.215 * *$ & $-.397 * *$ & $.47 * *$ & - \\
\hline 7.SFI & 7.05 & 1.46 & $.344 * *$ & $-.336^{* *}$ & $.437 * *$ & $.541 * *$ & $-.419 * *$ & $-.552 * *$ \\
\hline
\end{tabular}

$\mathrm{GHQ}=$ General Health Questionnaire, SFI $=$ Secure Flourishing Index, $* p<.05$. ** $p<.01$ correlated with experience of positive affect and flourishing ( $r s \geq .41, p s<.01)$ (see Table 1).

Further analyses revealed that male participants $(M=3.30, S D=.82)$ experienced a higher level of positive affect than female participants $(M=3.01, S D=.89)$, $t(388)=3.05, p=.002, d=.40,95 \%$ CI $[.1031, .4787]$. There was no gender differences on risk perception of COVID-19, self-efficacy in coping with COVID-19, experience of negative affect, severity of mental health issues, and experience of flourishing, $t s(388)<1.62$, $p s>.10$. Additional analyses revealed that married participants $(M=4.02$, $S D=.69)$ reported a higher level of self-efficacy in coping with COVID-19 than unmarried participants $(M=3.78$, $S D=.75), t(380)=3.27, p=.001, d=.33,95 \%$ CI [.0963, .3866]. Married participants also experienced a higher level of positive affect $(M=3.24, S D=.89$ vs. $M=2.93$, $S D=.83, t(380)=3.55, p<.001, d=.36,95 \%$ CI [.1399, .4872], a lower level of negative affect $(M=2.07, S D=.82$ vs. $M=2.29, S D=.93, t(380)=-2.47, p=.014, d=.25$, $95 \%$ CI $[.0453, .3982]$, and a higher level of flourishing than unmarried participants $(M=7.39, S D=1.41$ vs. $M=6.66$, $S D=1.42, t(380)=5.01, p<.001, d=.52,95 \%$ CI $[5.290$, 12.136].

\section{Predictors of Mental Health Problems and Flourishing during the COVID-19 Pandemic}

Next, we conducted a series of regression analyses to predict the severity of mental health problems and people's experience of flourishing during the COVID-19 pandemic. In the first regression analysis with sum score of the General Health Questionnaire as the dependent variable, and demographic variables, level of risk perception, self-efficacy, experience of negative and positive affect as predictors revealed that education level, experience of positive affect were negative predictors and experience of negative affect were positive predictors of the severity of mental health issues (see Table 2). Participants with a higher education level experienced a lower level of mental health issues during the COVID-19 pandemic. Those experienced a higher
Table 2 Predictors of mental health problems as measured by GHQ

\begin{tabular}{llllll}
\hline & $B$ & $S E$ & $\beta$ & $p$ & $95 \%$ CI \\
\hline Constant & 1.656 & 1.0 & & .099 & {$[-.310,3.622]$} \\
Age & .011 & .013 & .05 & .405 & {$[-.015, .037]$} \\
Gender & .355 & .202 & .072 & .080 & {$[-.042, .752]$} \\
Marital status & .302 & .207 & .082 & .146 & {$[-.106, .710]$} \\
Education & -.425 & .103 & -.169 & .000 & {$[-.629,-.222]$} \\
Risk perception & .193 & .154 & .057 & .210 & {$[-.109, .495]$} \\
Self-efficacy & .051 & .146 & .016 & .727 & {$[-.236, .338]$} \\
Positive affect & -.912 & .119 & -.351 & .000 & {$[-1.146,-.677]$} \\
Negative affect & 1.188 & .113 & .459 & .000 & {$[.967,1.410]$} \\
\hline
\end{tabular}

$\mathrm{GHQ}=$ General Health Questionnaire

Table 3 Predictors of flourishing as measured by the Secure Flourishing Measure

\begin{tabular}{llllll}
\hline & $B$ & $S E$ & $\beta$ & $p$ & $95 \%$ CI \\
\hline Constant & 4.157 & .588 & & .000 & {$[3.001,5.313]$} \\
Age & .008 & .008 & .057 & .297 & {$[-.007, .023]$} \\
Gender & -.05 & .119 & -.016 & .673 & {$[-.283, .183]$} \\
Marital status & .064 & .122 & .027 & .601 & {$[-.176, .304]$} \\
Education & .264 & .061 & .126 & .001 & {$[.085, .324]$} \\
Risk perception & -.149 & .09 & -.069 & .099 & {$[-.327, .027]$} \\
Self-efficacy & .345 & .086 & .173 & .000 & {$[.176, .514]$} \\
Positive affect & .643 & .07 & .387 & .000 & {$[.506, .781]$} \\
Negative affect & -.522 & .066 & -.314 & .000 & {$[-.652,-.392]$} \\
\hline
\end{tabular}

level of negative affect reported a higher level of mental health issues whereas those experience a higher level of positive affect reported a lower level of mental health issues. These results suggest that experience of negative affect is a risk factor whereas higher education level and experience of positive affect are protective factors of mental health during the COVID-19 pandemic.

Similarly, a second regression analysis with secure flourishing index as the dependent variable and demographic variables, level of risk perception, self-efficacy, experience of 
positive and negative affect as predictors revealed that education level, self-efficacy, and experience of positive affect were positive predictors and experience of negative affect was a negative predictor of flourishing (see Table 3 ). These results suggested that those received a higher level of education experienced a higher level of flourishing, which could be due to that those with a higher level of education level also have more financial and social resources to safeguard themselves from the negative impacts of the COVID-19 pandemic. Those who displayed a higher level of self-efficacy might adopt more adaptive coping strategies in response to the COVID-19 pandemic, and in turn, enhanced experience of flourishing during the pandemic. Consistent with the results on mental health issues, the results suggest that experience of positive affect is a protective factor whereas experience of negative affect was a risk factor for people's health and wellbeing as measured by the comprehensive measure of health - the flourishing measure.

\section{Mediation Analyses}

To further understand the role of positive and negative affect on the relationship between risk perception and severity of mental health problems and experience of flourishing, we conducted two mediational analyses by using Preacher and Hayes's (2008) indirect macro. First, we conducted a mediational analysis with level of risk perception as the independent variable, severity of mental health issues as the dependent variable, and experience of positive and negative affect as the mediating variables. The indirect effect of risk perception on severity of mental health problems through experience of positive and negative affect was significant $(B=.912, p<.001)$ (see Fig. 1). Bootstrapping analysis revealed that the $95 \%$ bias-corrected $C I$ for positive affect $[.1011, .3752]$ and negative affect $[.2714, .6566]$ did not include zero. The Sobel tests confirmed the mediation effect by positive affect $(Z=3.64, p<.001)$ and negative affect $(Z=5.44, p<.001)$. Therefore, experience of positive

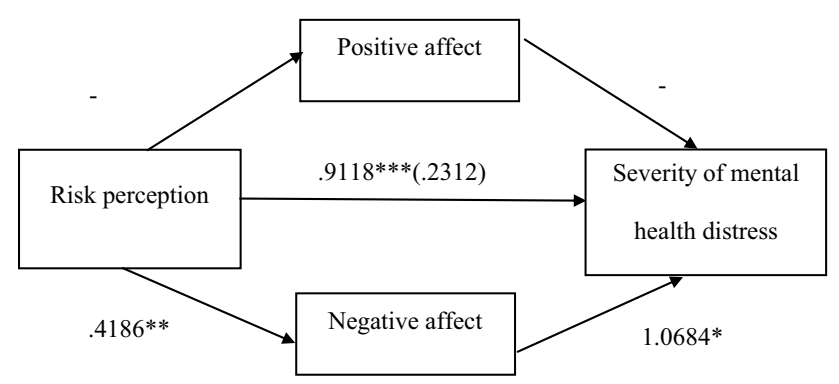

Fig. 1 Mediational model of positive and negative affect on risk perception and severity of mental health problems (GHQ), indirect effect was presented in parentheses. Note. Parameter estimates are unstandardized regression coefficients. $* * * p<.001$ and negative affect significantly mediated the relationship between risk perception and self-reported severity of mental health problems during the peak period of the COVID-19 pandemic in China.

Similarly, we conducted another mediational analysis with level of risk perception as the independent variable, experience of flourishing as the dependent variable, and experience of positive and negative affect as the mediating variables to understand the underlying mechanisms for the impact of risk perception on experience of flourishing. The indirect effect of risk perception on flourishing through experience of positive and negative affect was significant $(B=-.7298, p<.001)$ (see Fig. 2). Bootstrapping analysis revealed that the $95 \%$ bias-corrected CI for positive affect $[-.3343,-.0925]$ and negative affect $[-.3420,-.1367] \mathrm{did}$ not include zero. The Sobel tests confirmed the mediation effect by positive affect $(Z=-3.87, p<.001)$ and negative affect $(Z=-5.13, p<.001)$. Therefore, experience of positive and negative affect significantly mediated the relationship between risk perception and experience of flourishing during the peak period of the COVID-19 pandemic in China.

\section{Discussion}

The current research focuses on understanding the relationship between risk perception, emotional experiences, mental health, and flourishing during the peak period of the COVID-19 pandemic in China. Male participants, in general, reported a higher level of positive affect than female participants. The results demonstrated that higher education level and experience of positive affect were protective factors, whereas risk perception and experience of negative affect were risk factors, of mental health and flourishing. Furthermore, experience of positive and negative affect significantly mediated the relationship between risk perception and severity of mental health problems and experience of flourishing.

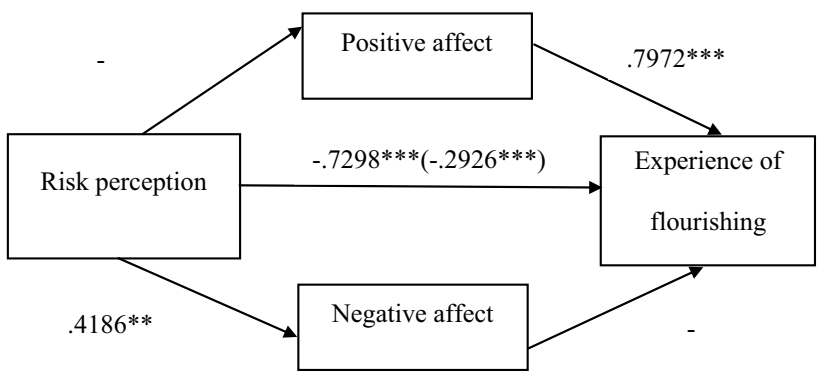

Fig. 2 Mediational model of positive and negative affect on risk perception and experience of flourishing (SFI), indirect effect was presented in parentheses. Note. Parameter estimates are unstandardized regression coefficients. $* * * p<.001$ 
Comparison between subgroups of the sample demonstrated that married participants reported a higher level of self-efficacy in coping with COVID-19, a higher experience of positive affect and flourishing, and a lower level of negative affect than unmarried participants. This suggest that marriage is a protective factor for coping with the mental health impact of the COVID-19 pandemic, which aligns with previous research on the factors that influence subject wellbeing (Diener et al., 1999) and our recent research on mental health of pharmacists during the COVID-19 pandemic in China. The result is also in line with recent research suggesting that married people value domains of well-being more than those who are single (Lee et al., 2020). One reason that married people reported a higher level of self-efficacy and mental well-being could be that married people have more financial and social resources available for them to cope with the negative impacts of COVID-19 pandemic than those who are unmarried. Another possibility is that married people are more connected with their family members and received more social support from their families during massive quarantine of the COVID-19 pandemic. Future research on marital status, social support, and mental health in times of public health crisis is needed to further verify this prediction.

The current research enriches our understanding on the relationship between risk perception of the COVID-19 pandemic and mental health by including both the negative (experience of mental health issues as measured by GHQ) and positive (experience of flourishing as measured by the flourishing measure) index of mental health. The General Health Questionnaire has been widely used as a convenient and sensitive measure of mental health illness (Goldberg et al., 1997). The flourishing index was developed recently as a comprehensive measure of health (VanderWeele, 2017; VanderWeele et al., 2019). These two measures helped us to gain a complete picture of the mental health status among Chinese during the peak period of the COVID-19 pandemic in China. About 27.2\% of participants reported experiencing some level of mental health issues during the peak period of COVID-19 pandemic in China, this is in line with recent research documenting the prevalence of mental health problems among the general public in the early stage of the pandemic (Liu et al., 2020; Qiu et al., 2020) and social media surveillance studies which documenting a negative relationship between risk perception and negative emotions during the first 11 weeks of the pandemic in China (Hou et al., 2020; Li et al., 2020). Despite the negative impact of the pandemic on people's mental health, however, the results also demonstrated that people experienced a relatively high level of flourishing, indicating resiliency among people in coping with the COVID-19 pandemic. We hope that this comprehensive measure of health will inspire future research on developing effective interventions to improve mental health and well-being and enhance resiliency during emergent public health crises (Zhang et al., 2022).

The current research also contributes to our understanding on the role of affective experiences on meant health and well-being (as measured by GHQ and the flourishing measure) by demonstrating the mediating effects of positive and negative affect on the severity of mental health distress and experience of flourishing. The results that positive and negative affect completely mediated the relationship between risk perception and mental health distress confirmed experience of negative affect as a risk factor of mental health and verified the buffering effect of positive affect in coping with mental health distress in face of stressful life events (Congard et al., 2020; Fredrickson et al., 2003; Ong et al., 2006; Steptoe et al., 2009; Tugade \& Fredrickson, 2007). This complete mediation effect also highlighted the central role of emotional experiences during the pandemic in influencing people's mental health and the adaptive functions of positive affect in dealing with the mental health impacts of this emergent public health crisis. Given that prioritizing positivity by enhancing the experience of positive emotions can safeguard people from mental health distress (Catalino et al., 2014; Congard et al., 2020), the current research results have implications for developing brief, effective, and scalable interventions to decrease negative affect and increase positive affect during this public health crisis. Researchers already initiated trials on enhancing positive affect and reducing negative affect through brief online interventions by changing reappraisal styles for adaptive emotion regulation during the COVID-19 pandemic (Wang et al., 2020). The partial mediation effect of positive and negative affect in explaining the relationship between risk perception and flourishing suggest that emotional experiences are an important factor that influence people's experiences of flourishing but there are other factors that may influence people's flourishing experience, which are not captured in the current research. Future research is warranted to further understand the underlying mechanisms that explaining the relationship between risk perception and flourishing. Future research with longitudinal design or brief interventions to enhance positive affect and mitigate negative affect will help public health practitioners and policy makers to establish and implement timely, scalable, and effective intervention strategies to improve pandemic preparedness and enhance resiliency.

The current research also has several limitations. First, the cross-sectional design precludes causal inference about the relationship between variables. Although mediational analyses enriched our understanding on the relationship between risk perception, emotional experiences, and mental health issues and flourishing, future research with longitudinal or intervention study design will provide stronger evidence for practical applications. Second, although the participants 
were from 31 provinces and one special administrative area (SAR) of China, the sample was not a representative of the Chinese population, which reduced the generalizability of the results to the general population. Third, the current research captured people's experiences of mental health and flourishing during the peak period of the COVID-19 pandemic in China, the results may not be generalized to people's experiences in the period of regular pandemic prevention and control when only occasional cases of infection are observed. Given that human beings are likely need to coexist with the COVID-19 pandemic for a relatively long period of time, longitudinal research is needed to capture dynamic changes of mental health, well-being, and flourishing among people both during and after the COVID-19 pandemic.

\section{Conclusion}

Although a substantial group of people experience mental health problems during the COVID-19 pandemic in China, they also experienced a relatively high level of flourishing, indicating resiliency in response to the disruptions of the COVID-19 pandemic. Besides, people's emotional experiences mediated the impact of risk perception on their mental health and experiences of flourishing. The results highlighted the importance of adaptive emotional regulation for maintaining health during the COVID-19 pandemic. Interventions focusing on increasing positive affect and decreasing negative affect are promising strategies to mitigate the negative mental health impact and enhance recovery and resiliency during public health crises such as COVID-19.

Acknowledgements Thanks Yun Kou for his help in distributing the study and Anran Wang for his help in editing the format of an earlier version of the manuscript.

Author Contribution The author designed the study, collected and analysed the data, wrote and revised the manuscript for publication.

Funding This study was funded by Hundred Talents Program Research Initiation Fund from Zhejiang University, the Leading Innovative and Entrepreneur Team Introduction Program of Zhejiang(2019R01007), Zhejiang University special scientific research fund for COVID-19 prevention and control (2020XGZX046).

Data Availability The datasets generated during and/or analysed during the current study are available from the corresponding author on reasonable request.

\section{Declarations}

Ethic Approval This study received ethic clearance from the author's hosted institution.
Consent to Participate Participants consented to participate in the study upon open the study link.

Consent for Publication The author consent to submit the manuscript for publication.

Conflict of Interest The author declares no conflicts of interests.

\section{References}

Banks, M. H., Clegg, C. W., Jackson, P. R., Kemp, N. J., Stafford, E. M., \& Wall, T. D. (1980). The use of the General Health Questionnaire as an indicator of mental health in occupational studies. Journal of Occupational Psychology, 53, 187-194. https://doi. org/10.1111/j.2044-8325.1980.tb00024.x

Brooks, S. K., Webster, R. K., Smith, L. E., Woodland, L., Wessely, S., Greenberg, N., \& Rubin, G. J. (2020). The psychological impact of quarantine and how to reduce it: Rapid review of the evidence. Lancet, 395(10227), 912-920. https://doi.org/10.1016/S01406736(20)30460-8

Catalino, L. I., Algoe, S. B., \& Fredrickson, B. L. (2014). Prioritizing positivity: An effective approach to pursuing happiness? Emotion, 14(6), 1155-1161. https://doi.org/10.1037/a0038029

Congard, A., Andreotti, E., Dauvier, B., Le Vigouroux, S., \& Antoine, P. (2020). Modification of affective trajectory in a positive psychology intervention. Applied Psychology: Health and WellBeing, 12(3), 770-786. https://doi.org/10.1111/aphw.12206

de Zwart, O., Veldhuijzen, I. K., Elam, G., Aro, A. R., Abraham, T., Bishop, G. D., Voeten, H. A., Richardus, J. H., \& Brug, J. (2009). Perceived threat, risk perception, and efficacy beliefs related to SARS and other (emerging) infectious diseases: Results of an international survey. International Journal of Behavioral Medicine, 16(1), 30-40. https://doi.org/10.1007/s12529-008-9008-2

Diener, E., Suh, E. M., Lucus, R. E., \& Smith, H. L. (1999). Subjective well-being: Three decades of progress. Psychological Bulletin, 125(2), 276-302. https://doi.org/10.1037/0033-2909.125.2.276

Folkman, S., \& Moskowitz, J. T. (2000). Stress, positive emotion, and coping. Current Directions in Psychological Science, 9(4), 115-118. https://doi.org/10.1111/1467-8721.00073

Fredrickson, B. L. (1998). What good are positive emotions? Review of General Psychology, 2, 300-319. https://doi.org/10.1037/10892680.2.3.300

Fredrickson, B. L. (2001). The role of positive emotions in positive psychology: The broaden-and-build theory of positive emotions. American Psychologist, 56, 218-226. https://doi.org/10.1037/ 0003-066x.56.3.218

Fredrickson, B. L. (2004). The broaden-and-build theory of positive emotions. Philosophical transactions of the Royal Society of London. Series B, Biological sciences, 359(1449), 1367-1378. https:// doi.org/10.1098/rstb.2004.1512

Fredrickson, B. L., Tugade, M. M., Waugh, C. E., \& Larkin, G. R. (2003). What good are positive emotions in crises? A prospective study of resilience and emotions following the terrorist attacks on the United States on September 11th, 2001. Journal of Personality and Social Psychology, 84(2), 365-376. https://doi.org/10.1037// $0022-3514.84 .2 .365$

Gan, Y. (2020). Happy people live longer and better: Advances in research on subjective well-being. Applied Psychology: Health and Well-Being, 12(1), 3-6. https://doi.org/10.1111/aphw.12192 
Goldberg, D. P., \& Hillier, V. F. (1979). A scaled version of the general health questionnaire. Psychological Medicine, 9, 139-145. https:// doi.org/10.1017/s0033291700021644

Goldberg, D., \& Williams, P. (1988). A user's guide to the general health questionnaire. NFER-Nelson.

Goldberg, D. P., Gater, R., Satorius, N., Üstün, T. B., Piccinelli, M., Gureje, O., \& Rutter, M. (1997). The validity of two versions of the GHQ in the WHO study of mental illness in general health care. Psychological Medicine, 27, 191-197. https://doi.org/10. 1017/s0033291796004242

Han, Q., Zheng, B., Agostini, M., Bélanger, J. J., Gützkow, B., Kreienkamp, J., Reitsema, A. M., van Breen, J. A., Collaboration, P., \& Leander, N. P. (2021). Associations of risk perception of COVID19 with emotion and mental health during the pandemic. Journal of Affective Disorders, 284, 247-255. https://doi.org/10.1016/j. jad.2021.01.049

Hou, Z., Du, F., Jiang, H., Zhou, X. \& Lin, L. (2020). Assessment of Public Attention, Risk Perception, Emotional and Behavioural Responses to the COVID-19 Outbreak: Social Media Surveillance in China. https://doi.org/10.2139/ssrn.3551338

Iorfa, S. K., Ottu, I., Oguntayo, R., Ayandele, O., Kolawole, S. O., Gandi, J. C., Dangiwa, A. L., \& Olapegba, P. O. (2020). COVID19 knowledge, risk perception, and precautionary behavior among Nigerians: A moderated mediation approach. Frontiers in Psychology, 11, 566773. https://doi.org/10.3389/fpsyg.2020.566773

John Hopkins University (2020) COVID-19 dashboard by the center for systems science and engineering (CSSE) at John Hopkins University (JHU). https://coronavirus.jhu.edu/map.html. Accessed 17 Mar 2021.

Lee, M. T., Bialowolski, P., Weziak-Bialowolska, D., Mooney, K. D., Lerner, P. J., McNeely, E., \& VanderWeele, T. J. (2020). Selfassessed importance of domains of flourishing: Demographics and correlations with well-being. The Journal of Positive Psychology. https://doi.org/10.1080/17439760.2020.1716050

Li, X., \& Lyu, H. (2021). Epidemic risk perception, perceived stress, and mental health during COVID-19 pandemic: A moderated mediating model. Frontiers in Psychology, 11, 563741. https:// doi.org/10.3389/fpsyg.2020.563741

Li, S., Wang, Y., Xue, J., Zhao, N., \& Zhu, T. (2020). The impact of COVID-19 epidemic declaration on psychological consequences: A study on active Weibo users. International Journal of Environmental Research and Public Health, 17(6), 2032. https://doi.org/ 10.3390/ijerph17062032

Liu, S., Yang, L., Zhang, C., et al. (2020). Online mental health services in China during the COVID-19 outbreak. The Lancet Psychiatry, 7(4), e17-e18. https://doi.org/10.1016/S2215-0366(20) 30077-8

Liu, C., Huang, N., Fu, M., Zhang, H., Feng, X. L., \& Guo, J. (2021). Relationship between risk perception, social support, and mental health among general Chinese population during the COVID-19 pandemic. Risk Management and Healthcare Policy, 14, 1843 1853. https://doi.org/10.2147/RMHP.S302521

Lötsch, F., Schnyder, J., Goorhuis, A., \& Grobusch, M. P. (2017). Neuropsychological long-term sequelae of Ebola virus disease survivors - A systematic review. Travel Medicine and Infectious Disease, 18, 18-23. https://doi.org/10.1016/j.tmaid.2017.05.001

Maunder, R., Hunter, J., Vincent, L., Bennett, J., Peladeau, N., Leszcz, M., Sadavoy, J., Verhaeghe, L. M., Steinberg, R., \& Mazzulli, T. (2003). The immediate psychological and occupational impact of the 2003 SARS outbreak in a teaching hospital. CMAJ : Canadian Medical Association Journal, 168(10), 1245-1251.

Maunder, R. G., Lancee, W. J., Balderson, K. E., Bennett, J. P., Borgundvaag, B., Evans, S., Fernandes, C. M., Goldbloom, D. S., Gupta, M., Hunter, J. J., McGillis Hall, L., Nagle, L. M., Pain, C., Peczeniuk, S. S., Raymond, G., Read, N., Rourke, S. B., Steinberg, R. J., Stewart, T. E., et al. (2006). Long-term psychological and occupational effects of providing hospital healthcare during SARS outbreak. Emerging Infectious Diseases, 12(12), 1924-1932. https://doi.org/10.3201/eid1212.060584

Ong, A. D., Bergeman, C. S., Bisconti, T. L., \& Wallace, K. A. (2006). Psychological resilience, positive emotions, and successful adaptation to stress in later life. Journal of Personality and Social Psychology, 91(4), 730-749. https://doi.org/10.1037/0022-3514. 91.4.730

Qiu, L., Zheng, X., \& Wang, Y. F. (2008). Revision of the positive and negative affect scale. Applied Psychology, 14(3), 249-254.

Qiu, J., Shen, B., Zhao, M., et al. (2020). A nationwide survey of psychological distress among Chinese people in the COVID19 epidemic: Implications and policy recommendations. General Psychiatry, 33, e100213. https://doi.org/10.1136/ gpsych-2020-100213

Steptoe, A., Dockray, S., \& Wardle, J. (2009). Positive affect and psychobiological processes relevant to health. Journal of Personality, 77(6), 1747-1776. https://doi.org/10.1111/j.1467-6494.2009. 00599.X

Tan, B. Y. Q., Chew, N. W. S., Lee, G. K. H., Jing, M., Goh, Y., Yeo, L. L. L., et al. (2020). Psychological impact of the COVID-19 pandemic on health care workers in Singapore. Annals of Internal Medicine. https://doi.org/10.7326/M20-1083

Tian, F., Li, H., Tian, S., Yang, J., Shao, J., \& Tian, C. (2020). Psychological symptoms of ordinary Chinese citizens based on SCL-90 during the level I emergency response to COVID-19. Psychiatry Research, 288, 112992. https://doi.org/10.1016/j.psychres.2020. 112992

Tugade, M. M., \& Fredrickson, B. L. (2007). Regulation of positive emotions: Emotion regulation strategies that promote resilience. Journal of Happiness Studies, 8, 311-333. https://doi.org/10. 1007/s10902-006-9015-4

VanderWeele, T. J. (2017). On the promotion of human flourishing. Proceedings of the National Academy of Sciences of USA, 114(31), 8148-8156. https://doi.org/10.1073/pnas.1702996114

VanderWeele, T. J., McNeely, E., \& Koh, H. K. (2019). Reimagining health-Flourishing. JAMA, 321(17), 1667-1668. https://doi.org/ 10.1001/jama.2019.3035

Wang, K., Goldenberg, A., Dorison, C., Miller, J., Lerner, J., Gross, J. \& Psychological Science Accelerator. (2020) A Global Test of Brief Reappraisal Interventions on Emotions During the COVID19 Pandemic. Nature Human Behaviour (forthcoming). 10.31234/ osf.io/m4gpq.

Watson, D., Clark, L. A., \& Tellegen, A. (1988). Development and validation of brief measures of positive and negative affect: The PANAS scales. Journal of Personality and Social Psychology, 54, 1063-1070. https://doi.org/10.1037/0022-3514.54.6.1063

Weziak-Białowolska, D., McNeely, E., \& VanderWeele, T. J. (2019). Human flourishing in cross-cultural settings: Evidence from the United States, China, Sri Lanka, Cambodia, and Mexico. Frontiers in Psychology, 10(29 May, 2019). https://doi.org/10.3389/ fpsyg.2019.01269.

Wise, T., Zbozinek, T. D., Michelini, G., Hagan, C. C., \& Mobbs, D. (2020). Changes in risk perception and self-reported protective behaviour during the first week of the COVID-19 pandemic in the United States. Royal Society Open Science, 7(9), 200742. https:// doi.org/10.1098/rsos.200742

Xiang, Y. T., Yang, Y., Li, W., Zhang, L., Zhang, Q., Cheung, T., \& $\mathrm{Ng}$, C. H. (2020). Timely mental health care for the 2019 novel coronavirus outbreak is urgently needed. The lancet. Psychiatry, 7(3), 228-229. https://doi.org/10.1016/S2215-0366(20)30046-8

$\mathrm{Xu}, \mathrm{Z}$., Wag, C., Liu, R., et al. (2013). Investigation on general mental health status and its influencing factors among adults in Jilin Province. Journal of Jilin University (Medical Edition), 39(6), 1282-1287. 
Y1ldırım, M., Arslan, G., \& Özaslan, A. (2020). Perceived risk and mental health problems among healthcare professionals during COVID-19 pandemic: Exploring the mediating effects of resilience and coronavirus fear. International Journal of Mental Health and Addiction, 1-11. Advance online publication. https:// doi.org/10.1007/s11469-020-00424-8.

Zhang, N., Wu, K., \& Wang, W. (2020). Timely mental health services contribute to the containment of COVID-19 pandemic in China. Global Health Research and Policy, 5(40). https://doi.org/10. 1186/s41256-020-00168-x

Zhang, N., Yang, H., Hong, D., Huang, X., \& Wang, L. (2021). Risk perception, self-efficacy, lay theories of health, and engagement in health-protective behaviors among hospital pharmacists during the COVID-19 pandemic. International Journal of Behavioral Medicine, 1-6. Advance online publication. https://doi.org/10. 1007/s12529-021-10004-2.
Zhang, N., Yang, S., \& Jia, P. (2022). Cultivating resilience during the COVID-19 pandemic: A socioecological perspective. Annual Review of Psychology. https://doi.org/10.1146/annur ev-psych-030221-031857

Publisher's Note Springer Nature remains neutral with regard to jurisdictional claims in published maps and institutional affiliations. 